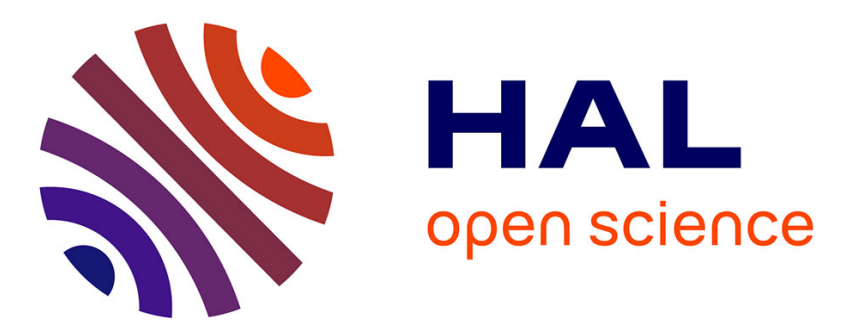

\title{
Dynamics of non-wetting drops confined in a Hele-Shaw cell
}

Ludovic Keiser, Khalil Jaafar, José Bico, Etienne Reyssat

\section{To cite this version:}

Ludovic Keiser, Khalil Jaafar, José Bico, Etienne Reyssat. Dynamics of non-wetting drops confined in a Hele-Shaw cell. Journal of Fluid Mechanics, 2018, 845, pp.245-262. 10.1017/jfm.2018.240 . hal-02104747

\section{HAL Id: hal-02104747 \\ https://hal.science/hal-02104747}

Submitted on 17 May 2019

HAL is a multi-disciplinary open access archive for the deposit and dissemination of scientific research documents, whether they are published or not. The documents may come from teaching and research institutions in France or abroad, or from public or private research centers.
L'archive ouverte pluridisciplinaire HAL, est destinée au dépôt et à la diffusion de documents scientifiques de niveau recherche, publiés ou non, émanant des établissements d'enseignement et de recherche français ou étrangers, des laboratoires publics ou privés. 


\title{
Dynamics of non-wetting drops confined in a Hele-Shaw cell
}

\author{
Ludovic Keiser ${ }^{1,2} \dagger$, Khalil Jaafar ${ }^{1}$, José Bico $^{1}$ and Etienne Reyssat ${ }^{1} \ddagger$ \\ ${ }^{1}$ Laboratoire de Physique et Mécanique des Milieux Hétérogènes (PMMH), CNRS, ESPCI \\ Paris, PSL Research University, 10 rue Vauquelin 75005 Paris, France; Sorbonne Université, \\ Univ. Paris Diderot \\ ${ }^{2}$ Total S.A., Pôle d'Études et de Recherche de Lacq, BP47 64170 Lacq, France
}

(Received xx; revised $\mathrm{xx}$; accepted $\mathrm{xx}$ )

We experimentally investigate the sedimentation of a non-wetting drop confined between two parallel walls. The whole system is immersed in a bath of liquid of low viscosity and a lubricating film may be dynamically formed between the drop and the walls of the cell. Depending on the thickness of the film and on the viscosity ratio between the droplet and the surrounding liquid, viscous dissipation localizes either in the lubrication layer or in the bulk of the drop. The velocity of the droplet is non-trivial as the thickness of the lubricating layer may depend on the interplay between interfacial tension and viscous dissipation. Interestingly, thin films whose nanometer thickness is set by long range intermolecular interactions may lubricate efficiently the motion of highly viscous droplets. We derive asymptotic models that successfully capture the settling velocity of the drop in the different regimes observed experimentally. The effect of partial wetting is finally illustrated by a sharp increase of the velocity of the drops that we attribute to a wetting transition.

\section{Introduction}

Droplet dynamics in confined media is a major issue for petroleum engineering as oil extraction commonly involves the displacement of microdroplets through pores of the same scale. The formation of such emulsions stems from various interfacial instabilities such as viscous fingering (Saffman \& Taylor 1958; Homsy 1987), splitting in the porous network (Jung et al. 2016), snap-off (Gauglitz \& Radke 1990; Dangla et al. 2013), or capillary instabilities in gradients of confinement (Pihler-Puzovic et al. 2012; AlHousseiny et al. 2012; Dias \& Miranda 2013; Keiser et al. 2016). The resulting multiphase flows generally involve large deformations of the interfaces (Zinchenko \& Davis 2017), which in turn modify the flow dynamics and make theoretical predictions of pressure losses complex. Droplet dynamics is however not limited to multiphase flows in porous media. Understanding the motion of a droplet in a confined environment is for instance crucial for digital microfluidics systems, which are currently blooming (Baroud et al. 2010; Seemann et al. 2012). Such confined droplets may even constitute a model system to describe the motion of biological cells in narrow environments (Zhou et al. 2007; Preira et al. 2013).

Numerous studies have been dedicated to model systems consisting of isolated droplets or bubbles moving in a uniform channel (Baroud et al. 2010). One of the fundamental questions concerns the peculiar viscous dissipation in the vicinity of menisci and contact lines (Snoeijer \& Andreotti 2013). Pioneering advances in the field were achieved by

$\dagger$ Email address for correspondence: ludovic.keiser@espci.org

$\ddagger$ Email address for correspondence: etienne.reyssat@espci.fr 
Landau \& Levich (1942) and Derjaguin (1993) who first described the coating of a plate withdrawn from a bath of wetting liquid. This mechanism is dictated by a balance of viscous stresses and surface tension. Bretherton (1961) adapted this problem to the case of a long bubble moving in a capillary tube filled with a wetting liquid. Later works extended this seminal study to the general case of viscous drops (Schwartz et al. 1986; Hodges et al. 2004). The recent development of digital microfluidic techniques has motivated numerous works on the dynamics of viscous drops confined in Hele-Shaw cells (Shen et al. 2014; Huerre et al. 2015; Zhu \& Gallaire 2016; Ling et al. 2016; Yahashi et al. 2016). A particular attention has been devoted to the lubricating role of the films of liquid separating the drop from the walls of the cell.

Eri \& Okumura (2011) investigated experimentally the settling of non-wetting drops in a Hele-Shaw cell, in the limit where viscous dissipation inside the drop dominates the resistance to the motion. Building up on these results, Yahashi et al. (2016) point out the crucial effect of lubricating films between the drop and the walls, in the regime of weakly confined drops. In the present paper, we explore experimentally and theoretically the effect of strong confinement and viscosity ratio between the drop and external liquids.

Drops of dense silicone oil sediment in a vertical Hele-Shaw cell filled with a solution of surfactant of lower viscosity. Depending on the confinement and on the contrast of viscosity, different flow regimes are observed as a function of the ability of the solution to lubricate the displacement of the droplet. In the following sections, we first present the experimental setup and describe the evolution of the sedimentation velocity as a function of the confinement and of the viscosity of the droplets in the ideal case where the surrounding liquid perfectly wets the wall of the cell. In the next section, we discuss these experimental data with different analytical models accounting for a self-lubrication effect. We then illustrate the case of partial wetting and demonstrate how the system may undergo a wetting transition with a dramatic impact on the droplet dynamics. We finally conclude and point out possible implications of our work for the study of the rheology of confined emulsions as encountered in petroleum engineering.

\section{Experiments}

\subsection{Experimental setup}

A Hele-Shaw cell made of two parallel glass slides separated by a gap of thickness $e$ ranging from 0.03 to $1 \mathrm{~mm}$ is filled with a surfactant solution and maintained vertically (Figure 1a). The solution is composed of $4.5 \%$ in mass of a commercial dishwashing soap (Paic Citron, from Colgate-Palmolive) in deionised water. This concentration is large in comparison with the critical micellar concentration of the soap ( $\mathrm{cmc} \simeq 0.05 \%$ in mass, determined with the standard Wilhelmy plate method). Such high concentrations should limit gradients in the local surfactant concentration at the interface and thus tend to hinder Marangoni effects. All experiments are conducted at room temperature $\left(20^{\circ} \mathrm{C}\right)$ for which the density and viscosity of the surfactant solution are $\rho_{w}=997 \mathrm{~kg} \cdot \mathrm{m}^{-3}$ and $\mu_{w}=1$ mPa.s, respectively.

A drop of fluorinated silicone oil (poly(3,3,3)-trifluoropropylmethylsiloxane, from Gelest, Inc.) is inserted into the gap of the cell. The oil density $\rho_{o}$ varies from 1220 to $1250 \mathrm{~kg} \cdot \mathrm{m}^{-3}$ depending on the selected viscosity $\mu_{o}$ ranging from 180 to $18000 \mathrm{mPa}$.s. The presence of surfactant molecules ensures a non-wetting condition for the oil droplet. In addition, while the interface between pure water and oil is very sensitive to contamination, the large excess of surfactant molecules also impose a reproducible value of the interfacial tension. The radius $R$ of the drop, typically millimetric, is larger than 


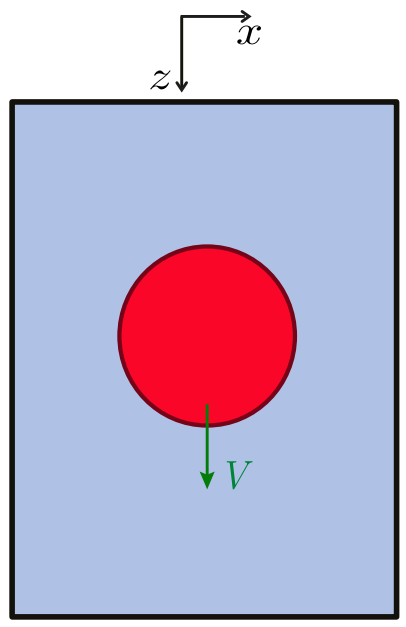

Front view

(a)

Side view

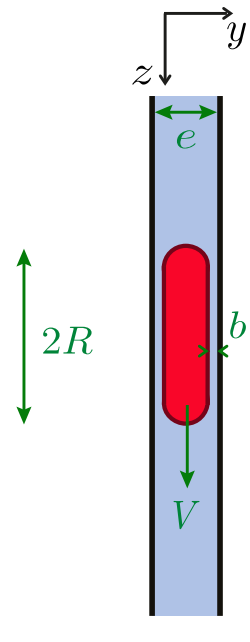

a)

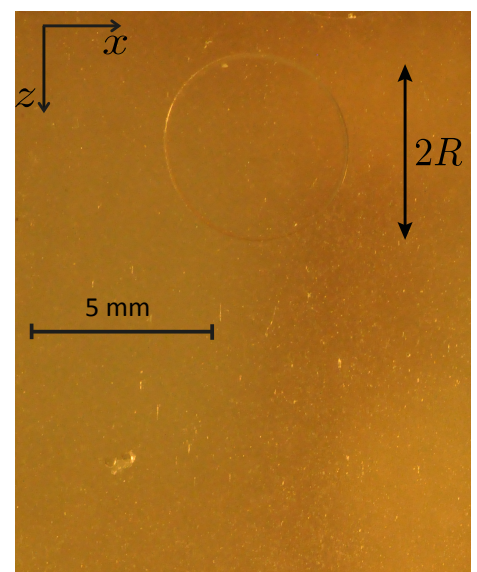

Front view

(b)

$V[\mathrm{~mm} / \mathrm{s}]$

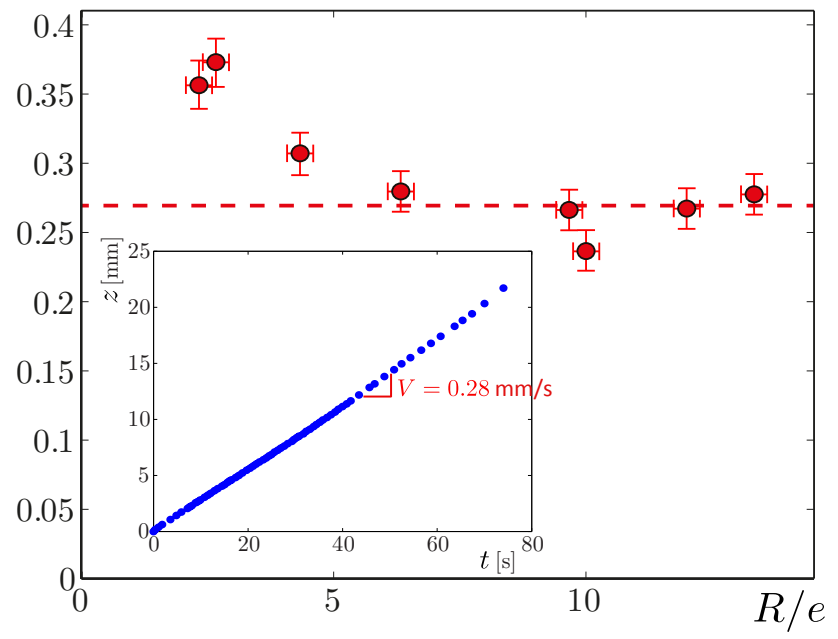

(c)

Figure 1. (a) Front and side views of the experimental setup: A droplet of fluorinated silicone oil of radius $R$ is introduced in a Hele-Shaw cell of inner gap $e$ immersed in a bath of surfactant solution, and sediments under its apparent weight. A thin layer of solution of thickness $b$ separates the drop from the walls of the cell. (b) Photograph representing the front view of a droplet (top of the picture) of radius $R \simeq 2.5 \mathrm{~mm}$ and of viscosity $\mu_{o}=1500 \mathrm{mPa} . \mathrm{s}$, moving down in a cell of gap thickness $e=400 \mu \mathrm{m}$. That circular shape has been observed for all droplets, regardless of their velocity. (c) Evolution of $V$ as a function of the ratio $R / e$, for $\mu_{o}=1500 \mathrm{mPa}$.s and $e=400 \mu \mathrm{m}$. For $R / e>5, V$ does not depend significantly on $R / e$. Inset: typical trajectory of a drop exhibiting a steady velocity (for $\mu_{o}=1500 \mathrm{mPa} . \mathrm{s}$ and $e=400 \mu \mathrm{m}$ ). 
$2 e$, so that the volume of these strongly confined puddles is approximately $\Omega=\pi R^{2} e$. As the surfactant solution totally wets the cell walls, a thin layer of solution of average thickness $b$ separates the drop from the wall. The water/oil interfacial tension $\gamma$ is measured with the sessile drop method and is between 5 and $6 \mathrm{mN} / \mathrm{m}$ depending on the oil viscosity. Under these conditions, the denser oil drop settles down under gravity at a constant velocity $V$ (see inset of Figure 1b). The sedimentation velocity weakly depends on the size of the drop. We interpret the weak dependence of the velocity as a result of geometry. While droplets adopt the shape of pancakes for high confinement, they tend to be spherical when $e \geqslant 2 R$. As a result, we do not observe any significant evolution for $R / e>5$. All experiments are thus conducted within this limit.

\subsection{Sedimentation velocity}

We represent in Figure 2 the evolution of the settling velocity $V$ of confined droplets as a function of the gap $e$ for various values of the oil viscosity $\mu_{o}$. Regardless of $\mu_{o}, V$ jumps by almost 5 orders of magnitude as $e$ increases from $30 \mu \mathrm{m}$ to $1 \mathrm{~mm}$.

Interestingly, $V$ appears very weakly dependent on $\mu_{o}$ for low values of $e(e<50 \mu \mathrm{m})$. In an intermediate regime $50 \mu \mathrm{m} \lesssim e \lesssim 700 \mu \mathrm{m}, V$ is a decreasing function of $\mu_{o}$. Finally, for $e \gtrsim 700 \mu \mathrm{m}$, the settling velocity becomes independent of the oil viscosity. The overall dependence of the drop velocity with its viscosity (see figure 2) is thus weaker than the simple inverse relationship that intuition might suggest. This result is in strong contrast with the opposite case of droplets that totally wet the walls (Reyssat 2014).

Different regimes are observed as a function of the confinement. Two trends are obtained for the lowest gaps $(e \lesssim 500 \mu \mathrm{m})$. While the evolution of the settling velocity seems roughly proportional to $e$ for the highest viscosity, we observe a variation with $e^{2}$ for droplets of low viscosity. However, all data tend to collapse in the same curve for larger gaps. The dependence with the gap then follows $V \propto e^{6}$, which is particularly striking.

\section{Lubrication model}

The weak dependence of the velocity with the viscosity of the drops suggests that viscous dissipation occurs not only in the drop but also in the thin films of solution separating the droplet from the walls of the cell. The aim of the present section is to identify the lubricating role of these films in the droplet dynamics.

\subsection{General analytic expression for the drop velocity}

We assume that a confined drop has the shape of a thick disc of volume $\Omega=\pi R^{2} e$, since the film thickness $b$ is much smaller than the gap $e$. The apparent weight of this drop thus writes:

$$
F_{g}=\Delta \rho g \pi R^{2} e
$$

where $\Delta \rho=\left(\rho_{o}-\rho_{w}\right)$ and $g=9.81 \mathrm{~m} \cdot \mathrm{s}^{-2}$ is the gravitational acceleration. This driving force is balanced by friction forces acting at the surface of the drop. However, due to the large value of the viscosity ratio $\mu_{o} / \mu_{w}$, we disregard in the following derivations the drag induced by bulk flows of surfactant solution around the drop. We also neglect the friction in the peripheral menisci of surfactant solution around the drop. Both assumptions are justified in Appendix A. Those negligible friction forces may however become dominant in the dynamics of foams (Cantat 2013) or confined bubbles (Reyssat 2014), where the viscosity ratio is conversely very small. In our experiments, the remaining source of friction corresponds to viscous stresses on the walls of the cell over the area $2 \pi R^{2}$ covered 


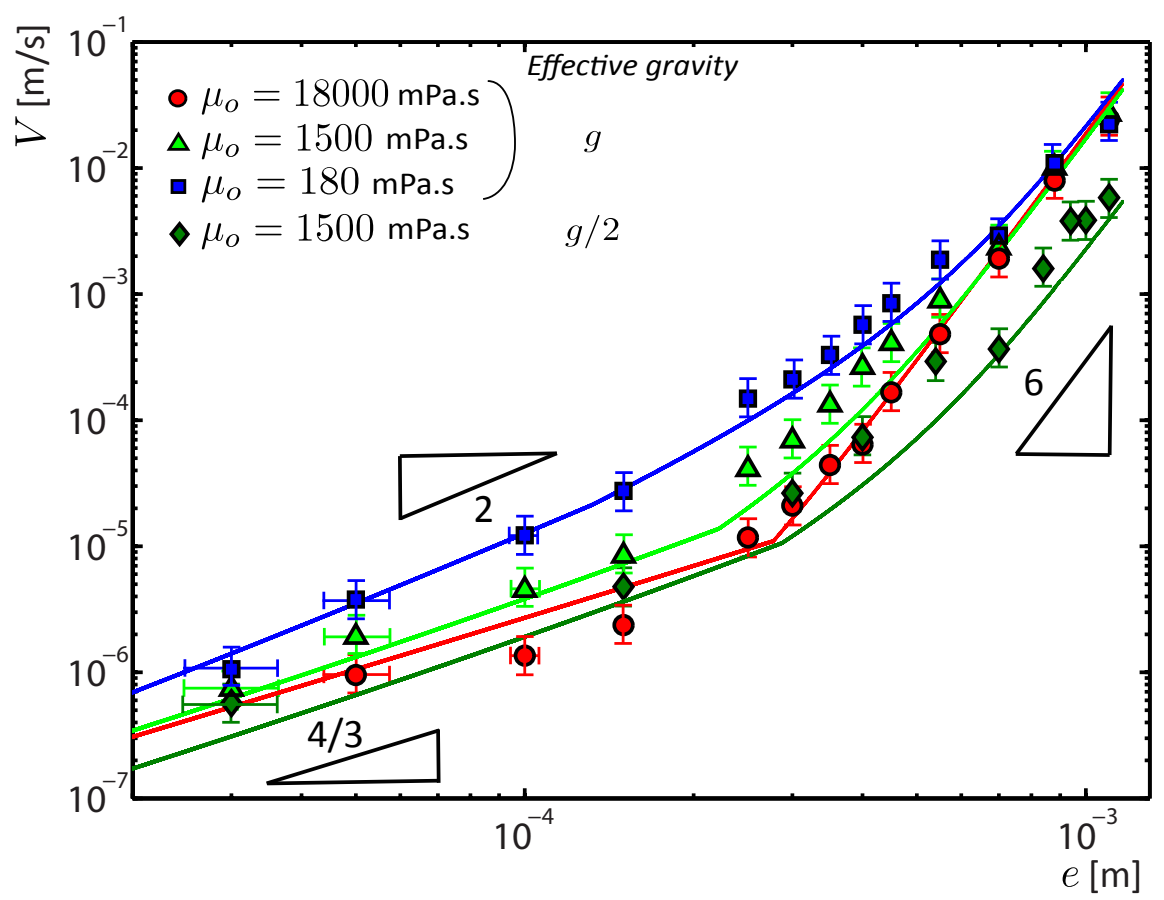

FiguRE 2. Evolution of the velocity of the confined drop $V$ as a function of the gap of the cell $e$. The blue squares, green triangles and red circles correspond respectively to $\mu_{o}=180 \mathrm{mPa}$.s, $\mu_{o}=1500 \mathrm{mPa} . \mathrm{s}$ and $\mu_{o}=18000 \mathrm{mPa}$.s. The dark green diamonds correspond to drops of viscosity $\mu_{o}=1500 \mathrm{mPa} . \mathrm{s}$ moving with a moderate gravity of magnitude $g / 2$, obtained by inclining the cell with an angle of $30^{\circ}$ with respect to the horizontal. The straight lines represent the resolution of the implicit Equation 3.19.

by the drop. Since both the driving force and the resistance are proportional to $R^{2}$, the droplet velocity should be independent of the size of the droplet as confirmed by the experiments. We expect this force balance to be valid for large drops, typically within the limit $R / e>5$ as illustrated in Figure 1. Smaller droplets are less confined by the wall, which changes their dynamics (they tend to move faster than larger ones). The drops are separated from the glass slides by a film of water whose average thickness $b$ is set dynamically by a balance between viscous and interfacial forces. As in the classic problem of a bubble penetrating in a capillary tube, we expect $b$ to be a function of the capillary number $C a_{w}=\mu_{w} V / \gamma$, where $\mu_{w}$ is the viscosity of water, $V$ the velocity of the drop and $\gamma$ the interfacial tension between the oil and the surfactant solution (Bretherton 1961). In our experiments, $C a_{w}$ varies between $10^{-7}$ and $10^{-2}$. However, in contrast with air bubbles, the viscosity of the drop $\mu_{o}$ cannot be neglected when compared with $\mu_{w}$. Indeed following Schwartz et al. (1986), $b$ should also slightly depend on the viscosity ratio $\mu_{o} / \mu_{w}$.

The velocity profile in a cross section of the Hele-Shaw cell is sketched in Figure 3a. We assume in the following derivations that the flow is directed along the direction of the average velocity $V$ and only varies across the section of the cell. We neglect the twodimensional component of the flow at the periphery of the drop, as well as transversal flows linked to the formation of "catamaran"-like dimples in the shape of the drops, as described by Huerre et al. (2015) and Zhu \& Gallaire (2016) . We finally define as $V_{\text {int }}$ the velocity at the interface, $y= \pm(e / 2-b)$, where $y=0$ corresponds to the center of the cell. 
We now derive the flow profile across the channel. In the region $|y|<(e / 2-b)$, the flow in the droplet follows a standard parabolic profile of average velocity $V$ :

$$
v_{d r o p}(y)=\frac{3}{2}\left(V-V_{i n t}\right)\left[1-\left(\frac{y}{e / 2-b}\right)^{2}\right]+V_{\text {int }}
$$

In the complementary region $e / 2-b<|y|<e / 2$, the thin lubricating film undergoes a simple shear flow with a no-slip boundary condition along the walls of the cell:

$$
v_{\text {film }}(y)=V_{\text {int }} \frac{e}{2 b}\left(\frac{2|y|}{e}-1\right)
$$

At the oil-film interface $(|y|=e / 2-b)$, the continuity of the tangential viscous stress in the drop and in the film imposes $\mu_{o} \frac{\partial v_{d r o p}}{\partial y}=\mu_{w} \frac{\partial v_{f i l m}}{\partial y}$. As $b \ll e$, this leads to:

$$
6 \mu_{o} \frac{V-V_{i n t}}{e}=\mu_{w} \frac{V_{i n t}}{b}
$$

We finally obtain for the velocity at the interface:

$$
V_{\text {int }}=\frac{V}{1+m}
$$

with $m=\mu_{w} e / 6 \mu_{o} b$. The dimensionless parameter $m$, quantifies the slipping of the drop with respect to the solid wall: while the limit $m \gg 1$ indicates very inefficient lubrication $\left(V_{\text {int }}=0\right), m \ll 1$ conversely corresponds to full slip $\left(V_{\text {int }}=V\right)$.

The shear stress exerted by the film of water and the resisting the motion of the drop is $\mu_{w} V_{i n t} / b$. Integrating this stress over the area $2 \pi R^{2}$ on both sides of the drop provides the friction force $F_{f}=2 \pi R^{2} \mu_{w} V_{i n t} / b$ acting on the drop. Inputting Equation 3.5 leads to:

$$
F_{f}=\frac{2 \pi R^{2} \mu_{w} V}{b(1+m)}
$$

We finally deduce the droplet velocity from the balance of $F_{f}$ with the apparent weight:

$$
V=\frac{\Delta \rho g e b}{2 \mu_{w}}(1+m)
$$

which may also be rewritten as:

$$
V=\frac{\Delta \rho g e^{2}}{12 \mu_{o}}\left(\frac{1}{m}+1\right)
$$

We now describe the asymptotic limits corresponding to $m \gg 1$ and $m \ll 1$, respectively.

\subsection{Inefficient lubrication: $m \gg 1$}

In the limit $m \gg 1$, the film does not play any significant role. Since $b \ll e$ the flow inside the droplet is described by a classical Poiseuille velocity profile:

$$
v(y)=\frac{3}{2} V\left(1-\left(\frac{2 y}{e}\right)^{2}\right)
$$

Integrating the resulting viscous stress over the interface of the drop gives $F_{f}=$ $12 \mu_{o} V \pi R^{2} / e$, which finally leads to the sedimentation velocity:

$$
V_{\text {Pois }}=\frac{\Delta \rho g}{12 \mu_{o}} e^{2}
$$



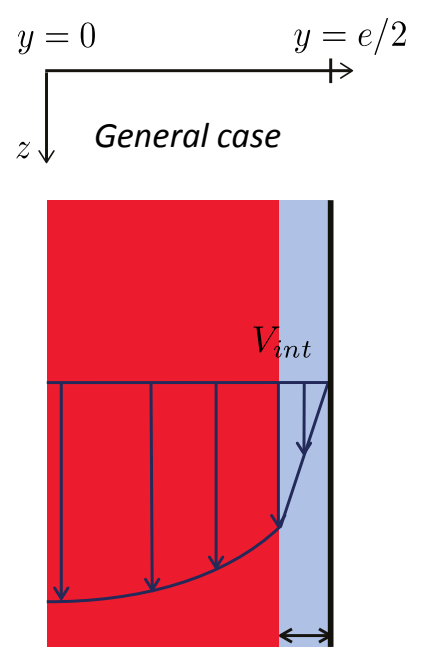

(a)

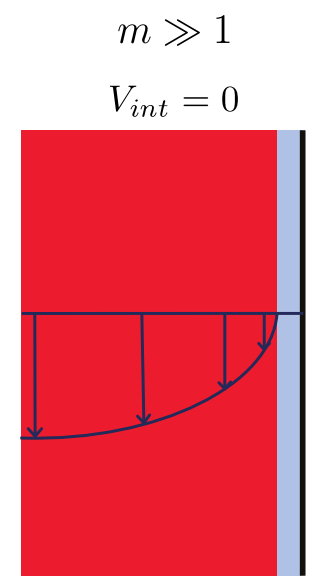

(b)

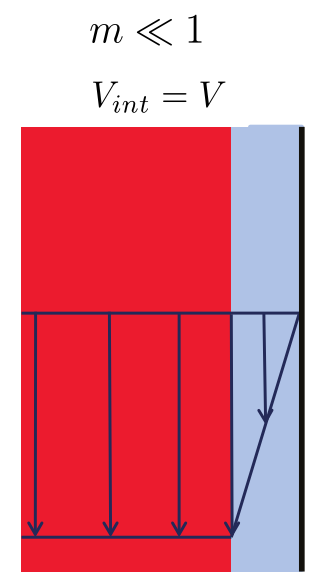

(c)

Figure 3. a) Velocity profile in a cross section of a half cell, for an intermediate value of the ratio $m=\mu_{w} e / 6 \mu_{o} b$. The origin of the $y$-coordinates is here taken in the middle of the gap, where the $z$-component of the velocity is maximal. b) Velocity profile in the limit $m \gg 1$. The film of water plays no lubricating role and the droplet undergoes a classical Poiseuille flow. c) Velocity profile in the limit $m \ll 1$. The drop moves as a solid plug and the film of water undergoes a Couette flow of shear rate $V / b$.

which can also be directly obtained by considering $m \gg 1$ in Equations 3.7. and 3.8. This prediction describes very well the experimental data corresponding to the oil of the lowest viscosity $\left(\mu_{o}=180 \mathrm{mPa} . \mathrm{s}\right)$ and small values of $C a_{w}$, i.e. high confinement (Figure 2), with no adjustable parameter (see also the dimensionless Figure 4). However, velocities measured with oils of higher viscosity or cells of larger gaps are higher than predicted in this asymptotic regime. In the following sections, we describe how the surrounding fluid can enhance the mobility of the droplet.

\subsection{Full lubrication: $m \ll 1$}

In the limit $m \ll 1$, the drop is simply translated as a solid block along the cell, while the lubrication film undergoes a Couette flow of shear rate $V / b$. The resisting force acting on the plug is thus given by:

$$
F_{f}=2 \pi R^{2} \frac{\mu_{w} V}{b}
$$

However, the thickness $b$ of the lubrication film should also depend on the dynamics. In his seminal work, Bretherton (1961) describes the film left behind a bubble moving in a capillary tube at velocity $V$. The thickness of the deposited film follows a nonlinear law: $b=1.34 r C a_{w}^{2 / 3}$, where $r$ is the radius of the tube and the capillary number, $C a_{w}=\mu_{w} V / \gamma$, which compares (thickening) viscous stresses with (thinning) surface tension. This law is in very good agreement with experimental results within the range $10^{-5} \lesssim C a_{w} \lesssim 10^{-2}$. At higher capillary numbers, the thickness of deposited the film tends to saturate as it becomes comparable with the radius of the tube (Taylor 1961; Aussillous \& Quéré 2000; Klaseboer et al. 2014). In the opposite limit of low $C a_{w}$, repulsive intermolecular interactions become dominant when the thickness of the film falls under a few hundreds of nanometers (Israelachvili 2011). Disjoining pressure may then stabilise nanometric films (Bergeron \& Radke 1992) and lead to a saturation of 
the coating thickness to a value $b_{\Pi}$ of the order of tens of nanometers, as first proposed by a referee of Bretherton's paper (Bretherton 1961). This effect was later modelled by Teletzke et al. (1988) and Chaudhury et al. (2014) and directly observed in a recent study by Huerre et al. (2015).

The work of Bretherton (1961) has also been extended to the motion of non-wetting viscous drops, which is relevant to our situation. Schwartz et al. (1986), Hodges et al. (2004) and more recently Balestra et al. (2017) have shown both theoretically and numerically that Bretherton's law should be corrected by a multiplying prefactor $P$ depending of the ratio $\mu_{o} / \mu_{w}$. Indeed, the finite viscosity of the drop amplifies viscous stresses and results into thicker films. In the range of experimental parameters corresponding to our experiment, $P$ should be close to $2^{2 / 3}$, which corresponds to the asymptotic value for very viscous drops $\left(\mu_{o} / \mu_{w} \rightarrow \infty\right)$, also derived by Bretherton.

Although the 2D geometry of the present problem is more complex than for the axisymmetric case of capillary tubes (Burgess \& Foster 1990; Reichert et al. 2018), we propose to extrapolate Bretherton's law to the case of droplets confined in a Hele-Shaw cell, as properly derived by Park \& Homsy (1984). The gap of the cell $e$ then replaces the radius $r$ of the capillary tube. However, the moving contact line is not perpendicular to the velocity, except at the apex of the drop. As a consequence, the film separating the drop of oil from the wall is not rigorously uniform, as shown experimentally by Huerre et al. (2015). However, we assume in the following that the average thickness of the lubricating layer is set by a relation of the form:

$$
b_{B r}=\alpha e C a_{w}^{2 / 3}
$$

where $\alpha$ is a numerical factor of the order of unity.

Nevertheless, we expect such a law to fail for low values of $C a_{w}$. In this limit, we assume $b$ to be equal to a value $b_{\Pi}$ dictated by the disjoining pressure in the film (Teletzke $e t$ al . 1988; Chaudhury et al. 2014). The actual value of $b_{\Pi}$ depends on the type of interactions involved in the disjoining pressure $\Pi_{D}$. In the case of ideal van der Waals forces, we expect the pressure in the film to be $\Pi_{D}=-A / 6 \pi b_{\Pi}^{3}$, where $A$ is the Hamaker constant (Israelachvili 2011). Balancing this pressure with the Laplace pressure $2 \gamma / e$ thus leads to:

$$
b_{\Pi}=\left(\frac{-A e}{12 \pi \gamma}\right)^{1 / 3}
$$

Considering $A \sim-10^{-20} \mathrm{~J}, \gamma \sim 5 \cdot 10^{-3} \mathrm{mN} / \mathrm{m}$ and $e \sim 50 \mu \mathrm{m}$, we obtain a typical estimate of $b_{\Pi} \sim 10 \mathrm{~nm}$. Nevertheless, molecular interactions are not limited to van der Waals forces. Other terms such as electrostatic repulsion may also play a role in the disjoining pressure and lead to other expressions for the film thickness. However, we expect the order of magnitude of $b_{\Pi}$ to remain on the order of a few tens on nanometers. Studying in detail the evolution of such thin films is beyond the scope of the current work. As detailed below, our experiment nevertheless constitutes a way to probe such thin films. We hope our study will motivate future investigations.

In the following sections we describe the regimes corresponding to both Bretherton and van der Waals limits.

\subsubsection{Bretherton film limit: $b>b_{\Pi}$ (self-lubrication)}

Inputting Bretherton's law in Equation 3.11 provides the friction force acting on the drop:

$$
F_{f}=\frac{\mu_{w}^{1 / 3} \gamma^{2 / 3} V^{1 / 3}}{\alpha e} 2 \pi R^{2}
$$


Interestingly, the friction force is a sub-linear function of the velocity, as discussed in more details at the end of the paper. Balancing this force with gravity, we obtain:

$$
V=\frac{\alpha^{3}}{8} \frac{(\Delta \rho g)^{3}}{\mu_{w} \gamma^{2}} e^{6}
$$

As mentioned earlier, $\alpha$ should be independent of the viscosity ratio, since $\mu_{o} / \mu_{w}>100$ in our experiments (Schwartz et al. 1986; Hodges et al. 2004; Balestra et al. 2017). Within this limit, $V$ should almost be independent of $\mu_{o}$ and be proportional to $e^{6}$ as observed experimentally (Figure 4). Fitting the experimental data with equation 3.15 provides an estimate of the numerical prefactor $\alpha=0.7 \pm 0.05$. This regime is very similar to the self-lubrication behavior observed in self-propelled droplets in capillary tubes (Bico \& Quéré 2002) and in sedimenting droplets in Hele-Shaw cells (Yahashi et al. 2016).

Rescaling Equation 3.15 by the velocity $V_{\text {Pois }}$ (Equation 3.10 ), we obtain the dimensionless velocity $\tilde{V}$ :

$$
\tilde{V}=\frac{3 \alpha^{3}}{2} \frac{\mu_{o}}{\mu_{w}}\left(\frac{e}{l_{c}}\right)^{4}
$$

This dependence of the rescaled velocity $\tilde{V}$ on $\left(e / \ell_{c}\right)^{4}$ may be observed on the dimensionless graph, on the Figure 4.

\subsubsection{Van der Waals film limit: low $C a_{w}$}

In this regime, $b=b_{\Pi}$ which leads to a friction force $F_{f}=2 \pi R^{2} \mu_{w} V / b_{\Pi}$. We thus expect the settling velocity of the droplet to be:

$$
V=\frac{\Delta \rho g b_{\Pi}}{2 \mu_{w}} e=\frac{\Delta \rho g}{2 \mu_{w}}\left(\frac{-A e^{4}}{12 \pi \gamma}\right)^{1 / 3}
$$

As in the previous regime, the velocity is independent of $\mu_{o}$, but is now proportional to $e^{4 / 3}$. Experimental data are in fair agreement with this prediction for strongly confined drops $(e<200 \mu \mathrm{m})$ of the highest viscosity, as illustrated in Figure 2. Adjusting Equation 3.17 leads to an estimation of the Hamaker constant $A \simeq-2 \cdot 10^{-20} \mathrm{~J}$. This evaluation of $A$ corresponds to thicknesses $b_{\Pi}$ ranging from 10 to $30 \mathrm{~nm}$ depending on the confinement, which is comparable to the values reported by Huerre et al. (2015). Rescaling Equation 3.17 by the velocity $V_{P o i s}$ (Equation 3.10 ), we obtain the dimensionless velocity $\tilde{V}$ for the van der Waals limit:

$$
\tilde{V}=\left(\frac{18}{\pi}\right)^{1 / 3} \frac{\mu_{o}}{\mu_{w}}\left(\frac{-A}{\gamma e^{2}}\right)^{1 / 3}
$$

\subsection{Configuration diagram}

The three regimes described in the previous section correspond to different asymptotic solutions of the equation:

$$
V=\frac{\Delta \rho g e}{2}\left(\frac{e}{6 \mu_{o}}+\frac{\max \left(b_{\Pi} ; b_{B r}\right)}{\mu_{w}}\right)
$$

where $b_{B r}$ and $b_{\Pi}$ are respectively defined by Eqs. 3.12 and 3.13. We arbitrarily assume that $b=\max \left(b_{\Pi} ; b_{B r}\right)$. Although the transition between both film regimes is actually smoother, we found this simple interpolation to be in good agreement with experiments. The implicit Equation 3.19 can be solved numerically and captures correctly the experimental data obtained for different values of viscosity ratio and effective gravity, as represented in Figure 2. 


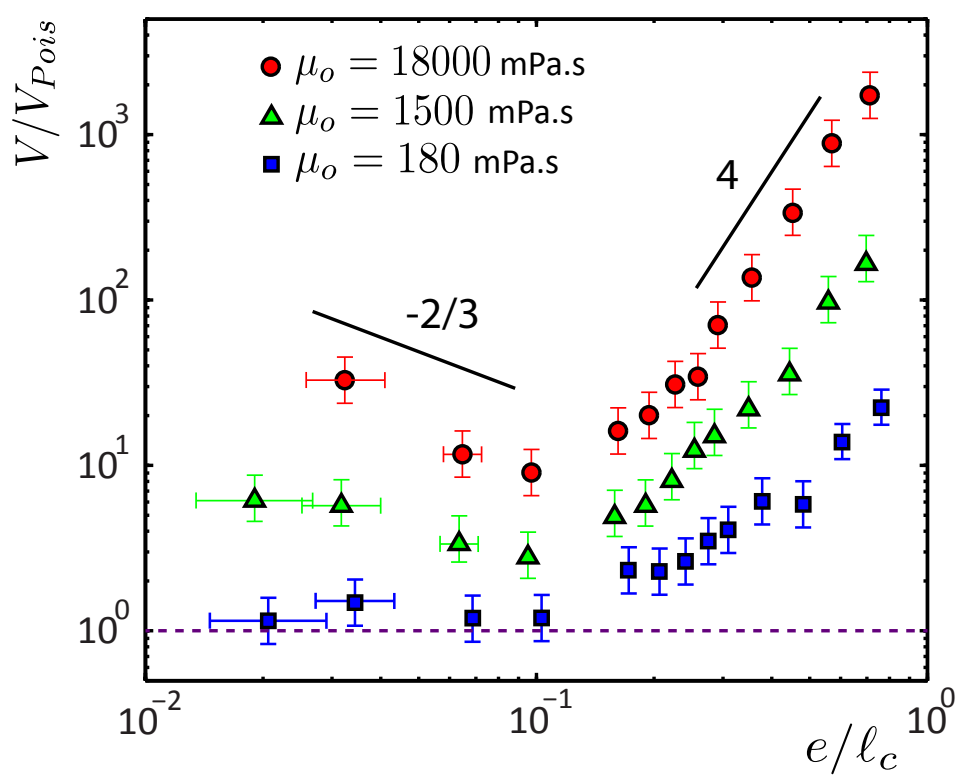

Figure 4. Dimensionless velocity $\tilde{V}=V / V_{P o i s}$ as a function of the rescaled gap thickness $e / \ell_{c}$, with $\ell_{c}$ the capillary length. The purple dashed line corresponds to the Poiseuille regime characterized by $\tilde{V}=1$. For most of the experimental measurements, the rescaled velocity $\tilde{V}$ is larger than unity, which enhances the lubricating role of the films of water between the drop and the walls.

Using equation 3.10, Equation 3.19 may be rewritten in a dimensionless implicit form:

$$
\tilde{V}=1+6 \frac{\mu_{o}}{\mu_{w}} \max \left(\left(\frac{-A}{12 \pi \gamma e^{2}}\right)^{1 / 3} ; \frac{\alpha}{12^{2 / 3}}\left(\frac{\mu_{w}}{\mu_{o}}\right)^{2 / 3}\left(\frac{e}{\ell_{c}}\right)^{4 / 3} \tilde{V}^{2 / 3}\right)
$$

In figure 4 we plot the dimensionless velocity $\tilde{V}$ as a function of confinement $e$ normalized by the capillary lenght $\ell_{c}$. The lubricating properties appear clearly here, as $\tilde{V} \geqslant 1$ for all data points. The drops of the most viscous oils $(\mu=18000 \mathrm{mPa} . \mathrm{s})$ are always fully lubricated, either by a Bretherton film $\left(\tilde{V} \propto e^{4}\right)$ or by a van der Waals film $\left(\tilde{V} \propto e^{-2 / 3}\right)$. Less viscous oils benefit less from the lubricating properties of water films. In particular, for oils less viscous than about $200 \mathrm{mPa} . \mathrm{s}$, the regime dominated by dissipation in the drop is clearly observed $(\tilde{V}=1$, blue squares in figure 4$)$.

Describing the problem in terms of configuration diagram may be useful to get a global view of the different regimes. For a given oil viscosity, two or three regimes may be observed as the gap is increased. For very high confinement the thin film of water acts as a lubricant and the settling velocity is proportional to $e^{4 / 3}$ in the case of an ideal van der Waals film, as defined in Equation 3.17. In the opposite limit, self lubrication plays a leading role and leads to $V$ proportional to $e^{6}$ as described by Equation 3.15. Between both regimes, the effect of the film may be negligible and the droplet undergoes a classical Poiseuille flow with a velocity varying with $e^{2}$ (Equation 3.10 ). We define as $e_{4 / 3 \rightarrow 2}$ and $e_{2 \rightarrow 6}$ the characteristic gaps at the transitions from the regimes $V \propto e^{4 / 3}$ to $V \propto e^{2}$ and $V \propto e^{2}$ to $V \propto e^{6}$, respectively. Both characteristic gaps are readily deduced by equating the corresponding velocities:

$$
\frac{e_{4 / 3 \rightarrow 2}}{\ell_{c}}=\left(\frac{\mu_{o}}{\mu_{w}}\right)^{3 / 2}\left(\frac{-18}{\pi} \frac{A}{\gamma \ell_{c}^{2}}\right)^{1 / 2}
$$


and

$$
\frac{e_{2 \rightarrow 6}}{\ell_{c}}=\left(\frac{2}{3 \alpha^{3}} \frac{\mu_{w}}{\mu_{o}}\right)^{1 / 4}
$$

where $\ell_{c}=(\gamma / \Delta \rho g)^{1 / 2}$ is defined as the capillary length, whose value is generally of the order of $1 \mathrm{~mm}$ (in our particular system, $\ell_{c} \simeq 1.5 \mathrm{~mm}$ ).

However a direct transition from the regime $V \propto e^{4 / 3}$ to $V \propto e^{6}$ can also be observed if $e_{4 / 3 \rightarrow 2}>e_{2 \rightarrow 6}$. This transition is characterized by the following gap:

$$
\frac{e_{4 / 3 \rightarrow 6}}{\ell_{c}}=\left(\frac{-16}{3 \pi \alpha^{9}} \frac{A}{\gamma \ell_{c}^{2}}\right)^{1 / 14}
$$

This direct transition is only observed for the highest viscosity ratios:

$$
\frac{\mu_{o}}{\mu_{w}}>\left(\frac{\pi^{2}}{486 \alpha^{3}} \frac{\gamma^{2} \ell_{c}^{4}}{A^{2}}\right)^{1 / 7}
$$

In our system, the numerical value of the right hand side of Equation 3.24 is of the order of 1650, which is in fair agreement with our experimental observations. Indeed we can clearly define two regimes $\left(V \propto e^{2}\right.$ and $\left.V \propto e^{6}\right)$ for $\mu_{o}=180 \mathrm{mPa}$.s, and two regimes $\left(V \propto e^{4 / 3}\right.$ and $\left.V \propto e^{6}\right)$ for $\mu_{o}=18000 \mathrm{mPa}$.s. However, the two low-velocity regimes $V \propto e^{4 / 3}$ and $V \propto e^{2}$ seem transitional in the intermediate case $\mu_{o}=1500 \mathrm{mPa} . \mathrm{s}$ (Figure 2). We present in Figure 5, the configuration diagram corresponding to these different transitions. In the case of high contrast of viscosity, the deposited film plays the role of a lubricant, which leads to settling velocities proportional to $e$ and $e^{6}$ for respectively strong and weak confinements. Conversely, dissipation is concentrated in the bulk of the droplet when the contrast in viscosity is modest, which results in a velocity proportional to $e^{2}$.

\section{Partially wetting drops: lubrication film properties recovered through a wetting transition}

In contrast with the ideal complete wetting situation described so far, practical configurations commonly involve partial wetting. In such cases, we do not expect to obtain any lubricating film below a certain value of $C a_{w}$ (Marchand et al. 2012), which should strongly affect the droplet dynamics. We performed a series of experiments where the solution of surfactant is replaced by ethanol. In this system, ethanol partially wets the walls of the cell with a receding contact angle of the order of $30^{\circ}$. The viscosity of ethanol is $\mu_{w}=1.2 \mathrm{mPa} . \mathrm{s}$ at $20^{\circ} \mathrm{C}$.

In Figure 6, we plot $V$ as a function of $e$ for drops of oil of respective viscosities $\mu_{o}=180,1500$ and $18000 \mathrm{mPa}$.s. At the lowest values of $e$ (most confined cells), $V$ tends to follow the regime described by Equation 3.10 (dashed lines), which corresponds to a Poiseuille flow without any lubrication. In contrast with the previous experiments conducted with a wetting solution, this regime is obtained with oils of all viscosities, which confirms the absence of a lubricating film. As the thickness of the cell is increased, we observe a jump in the velocity for a critical thickness. This jump is particularly dramatic for the most viscous oils: the velocity increases by two orders of magnitude for the oil of viscosity $18000 \mathrm{mPa}$.s. Beyond this critical thickness, the settling velocity follows the self-lubrication regime described by Equation 3.15. We interpret the jump as the consequence of a wetting transition: a lubricating film is deposited as $C a_{w}$ reaches a critical value. This critical velocity has been discussed in simpler geometries for a 


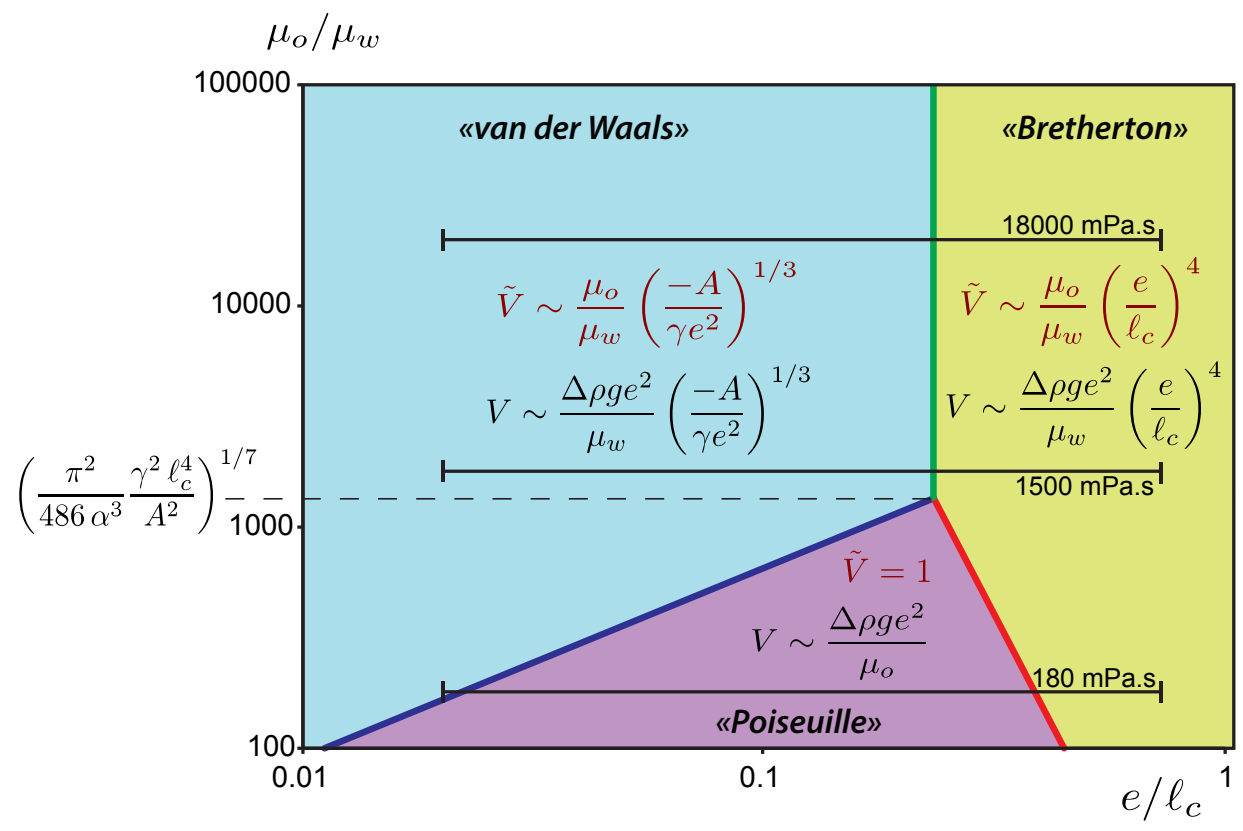

FiguRE 5. Configuration diagram describing the different asymptotic regimes as a function of the gap of the cell and of the viscosity ratio. The range of parameters explored in our experiments are represented with the horizontal lines corresponding to the different oil viscosities. Depending on the viscosity ratio, one or two transitions can be observed. The frontiers between each regime are represented by colourful lines. Blue, green and red lines correspond respectively to Equations $3.21,3.22$ and 3.23. In each domain, the asymptotic expression of the velocity $V$ corresponds to the scaling of Equations 3.17 (cyan domain), 3.15 (yellow domain) and 3.10 (magenta domain). The scaling of the rescaled expression $\tilde{V}=V / V_{\text {Pois }}$ is written in brown, and correspond respectively to Equations 3.18, 3.16 and by definition $\tilde{V}=1$.

variety of viscosity contrasts (Eggers 2001; Lorenceau et al. 2003; Snoeijer et al. 2006; Marchand et al. 2012). In the present case, the deposited fluid is much less viscous than the oil in the drops, which is reminiscent from the air entrainment problem. In this limit, Eggers and Lorenceau et al. found that the critical velocity for entrainment depends logarithmically on the viscosity ratio, while Marchand et al. rather suggest a stronger power-law dependence on the viscosity of air. However, in our experiments the component of the velocity normal to the contact line varies along the boundary of the drop (Burgess \& Foster 1990). This component decreases from the front to the sides of the drop, which should delay or smoothen the transition. We also observe an important scattering of the experimental data due to a high sensitivity to local imperfections. As a general trend, smaller drops tend to undergo the transition for lower gaps than larger drops. Following the steps of the recent works of Hammoud et al. (2017) and Zhao et al. (2018), a better characterization of this transition may be achieved in the axisymmetric geometry of a capillary tube.

\section{Conclusion}

Our experimental study describes the sedimentation velocity of a droplet of viscous oil in a Hele-Shaw cell filled with a less viscous solution $\left(\mu_{o} / \mu_{w} \gg 10\right)$. Depending on the confinement and on the wetting properties of the solution, different regimes are 


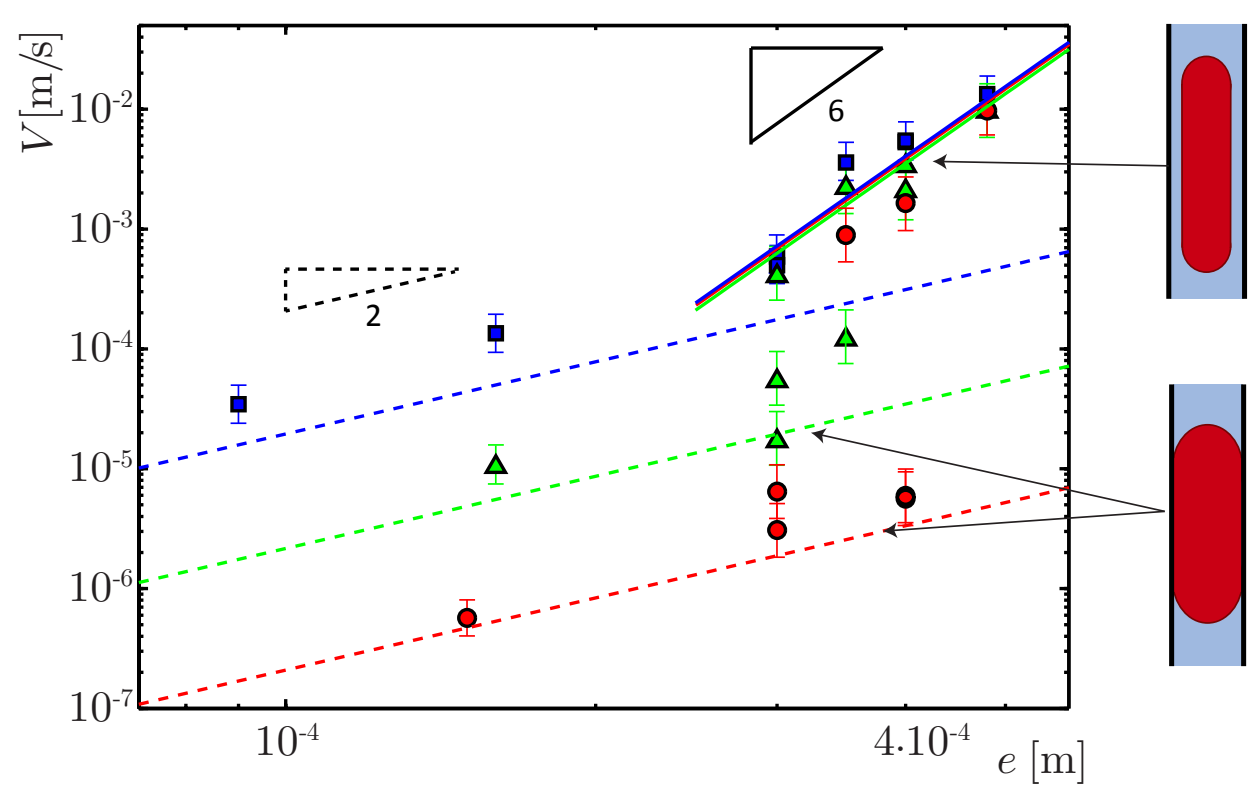

Figure 6 . Settling velocity $V$ as a function of the gap of the cell $e$ for partially wetting drops of oil in a cell filled with ethanol. Blue squares, green triangles and red circles correspond respectively to $\mu_{o}=180 \mathrm{mPa} . \mathrm{s}, \mu_{o}=1500 \mathrm{mPa} . \mathrm{s}$ and $\mu_{o}=18000 \mathrm{mPa} . \mathrm{s}$. We interpret the important jump observed beyond a critical gap to be a consequence of a wetting transition. The dotted lines correspond to the "Poiseuille regime" (equation 3.10) and the solid lines correspond to the "self-lubricated" regime with a Bretherton film (equation 3.15, with $\alpha=0.7$ )

observed. In particular, we demonstrated a possible self-lubrication effect of the droplet by the solution, which dramatically amplifies the sedimentation mobility. In the absence of lubrication, the sedimentation velocity scales as $\Delta \rho g e^{2} / \mu_{o}$. However, self-lubrication leads to a velocity proportional to $e^{6}$ in the case of low confinement and to a velocity proportional to $e^{4 / 3}$ for high confinements (in the case of a wetting solution). Although we mainly focused on the case of a perfectly wetting carrying liquid, we observe a stiff signature of a wetting transition in the case of partial wetting.

This work may also provide some insight into the rheology of dense emulsions in confined environments. Such a situation is indeed common in petroleum engineering when the size of oil droplets is of the same scale as the pores of the reservoirs. By analogy with standard rheology data, we can define an effective viscosity $\mu_{\text {eff }} \sim \Delta \rho g e^{2} / V$ and express it as a function of the apparent shear rate $V / e$. The three different regimes presented in Figure 5 correspond to the following expressions:

- Lubrication through the thin van der Waals film: $\mu_{e f f} \sim \mu_{w} e / b_{\Pi} \sim \mu_{w}\left(\frac{\gamma e^{2}}{-A}\right)^{1 / 3}$

- Absence of lubrication: $\mu_{e f f}=\mu_{o}$,

- Lubrication through the Bretherton film: $\mu_{e f f} \sim\left(\mu_{w} \gamma^{2} e^{2}\right)^{1 / 3}(V / e)^{-2 / 3}$.

If the apparent shear rate could be increased as a control parameter, the confined emulsion would thus appear as a shear thinning fluid. The effective viscosity is constant at low shear rate and displays a power law behavior with an exponent $-2 / 3$ for high shear rates. Such a behavior is reminiscent of classical works on the rheology of foams and concentrated emulsions, although dissipation in Plateau borders tends to lead to a lower exponent (Princen \& Kiss 1989; Denkov et al. 2008; Cantat 2013; Cohen-Addad \& Höhler 2014). 
In our model, we assumed the lubricating film to be uniform in order to simplify the description of the system, which is not rigorous as demonstrated experimentally by Huerre et al. (2015). Nevertheless, we hope that our work will motivate further investigations where the thickness profile and, more generally, the impact of the $2 \mathrm{D}$ geometry of the droplet will be taken into account.

Finally, our experiments demonstrate the critical role of the lubricating film of water in the dynamics of highly viscous drops. As a perspective, it might be expected that any variation of the thickness of that film, induced by e.g. rough (Seiwert et al. 2011) or slippery (Li et al. 2014) walls, could hinder the self-lubrication regime (equation 3.15) and thus dramatically modify the mobility of the drops.

\section{Acknowledgements}

We warmly thank Armelle Keiser for her original contribution, and Bertrand Levaché, Hélène Berthet and Maurice Bourrel for fruitful discussions. We are grateful to anonymous referees for their relevant and helpful suggestions. The financial support from Total S.A. and from the Interuniversity Attraction Poles Programme (IAP 7/38 MicroMAST) initiated by the Belgian Science Policy Office is gratefully acknowledged.

\section{Appendix A}

We here justify our choice to neglect the resisting contribution of the flow of water in the front and rear menisci as well as in the bulk around the drop.

\section{Dissipation in the bulk of water surrounding the drop}

The resisting force resulting from the flow of water around the drop scales as $F_{w}^{b u l k}=$ $12 \pi \mu_{w} V R^{2} / e$ which has to be compared to the friction force $F_{f}=2 \pi R^{2} \mu_{w} V_{i n t} / b$, exerted

by the walls on the drop. Using Equation 3.5, we obtain: $F_{w}^{b u l k} / F_{f}=6 b(1+m) / e$. As plotted in Figure $7 \mathrm{a}$, this ratio is at most $2.10^{-1}$ within the range of our experimental parameters. The contribution of viscous dissipation in the bulk of the surrounding water to the overall friction force can thus be neglected.

\section{Dissipation in the front and rear menisci}

Following recent work on the motion of wetting droplets in Hele-Shaw cells (Reyssat 2014 ) or on the dissipation involved in the displacement or deformation of foams (Cantat 2013; Sauret et al. 2015; Viola et al. 2016), we expect the friction force resulting from the flow in the advancing and receding menisci of water around the drop to follow $F_{w}^{m e n i s} \simeq 20 \gamma R C a_{w}^{2 / 3}$. Comparing Equation 3.6 with this force yields: $F_{w}^{m e n i s} / F_{f} \simeq$ $3 b(1+m) C a_{w}^{-1 / 3} / R$. As plotted in Figure $7 \mathrm{~b}$, this ratio is of the order of 0.2 for the largest gaps, but lower than 0.1 for more confined drops, which justifies that the contribution of the menisci may be neglected in the overall estimation of the friction.

\section{Inertial resistance of water}

The inertial resistance of the displaced water also hinders the motion of the drop and scales as $F_{i} \sim \rho_{w} V^{2} R e$. The comparison of inertia to the friction force acting on the surface of water written in Equation 3.6 leads to $F_{i} / F_{f}=\rho_{w} V b e(1+m) / \mu_{w} R$, which can be viewed as the relevant Reynolds number. For our experiments, this Reynolds number remains smaller than 1 as long as $e<1 \mathrm{~mm}$, which justifies neglecting the contribution 

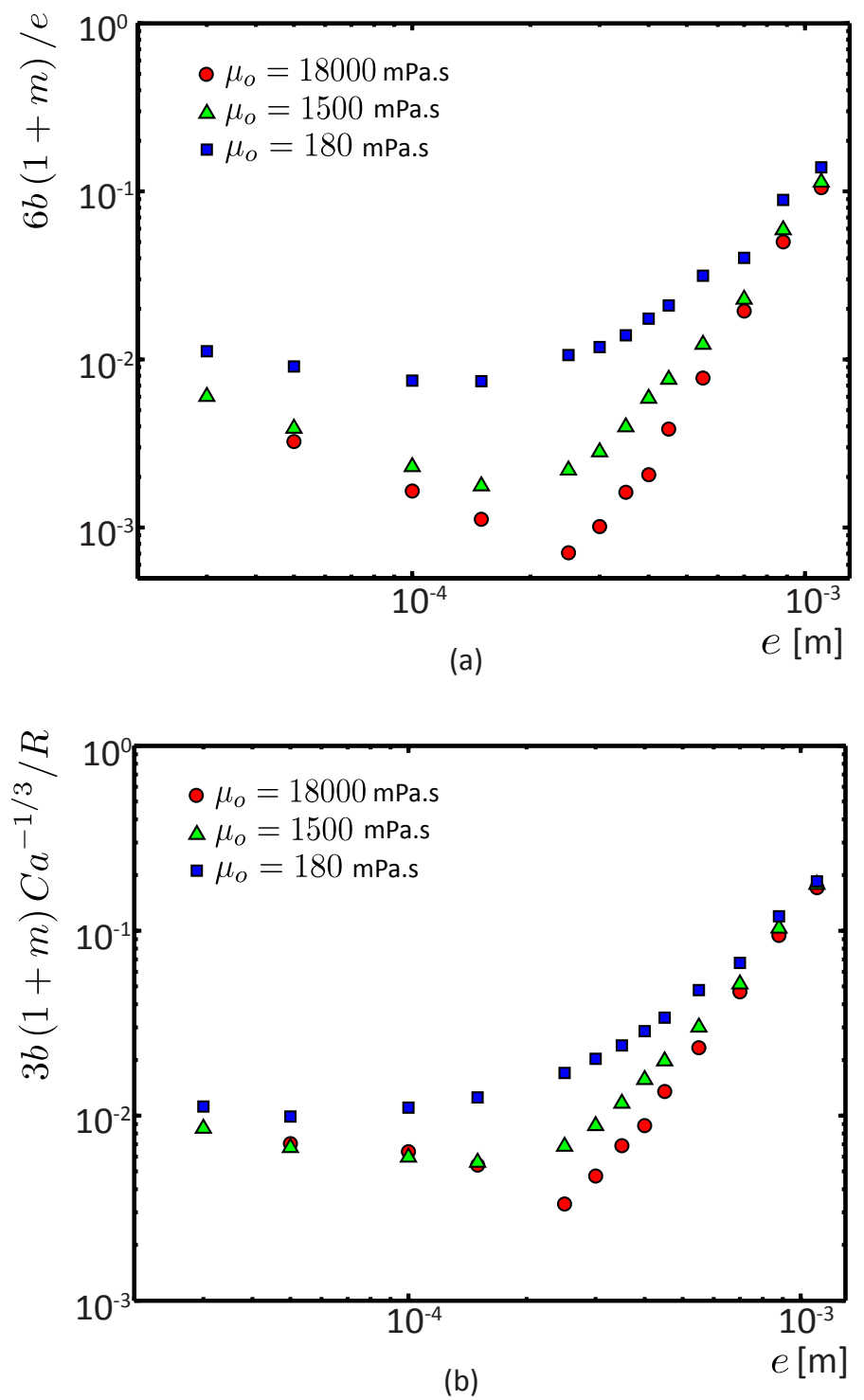

Figure 7. (a) Ratio of the viscous force from the bulk of the surrounding liquid to the force exerted by the lubrication film as a function of $e$. Blue squares correspond to $\mu_{o}=180 \mathrm{mPa}$.s, green triangles to $\mu_{o}=1500 \mathrm{mPa} . \mathrm{s}$, and red circles to $\mu_{o}=18000 \mathrm{mPa}$.s. The ratio roughly corresponds to $6 b(1+m) / e$, and its value is smaller than 0.2 for all the experiments, so that the contribution of the surrounding liquid is negligible. (b) Ratio of the viscous force in the menisci at the periphery of the drop to the force from the lubrication film as a function of $e$. $F_{w}^{\text {menis }} / F_{f} \simeq 3 b(1+m) C a_{w}^{-1 / 3} / R$, is smaller than 0.2 for all the experiments and the contribution of the menisci may be neglected.

of inertia in the fluid surrounding the drop in the overall drag force. For $e>1 \mathrm{~mm}$ the drop velocity is observed to saturate as inertial resistance starts to dominate. 


\section{REFERENCES}

Al-Housseiny, T. T., Tsai, P. A. \& Stone, H. A. 2012 Control of interfacial instabilities using flow geometry. Nature Physics 8 (10), 747-750.

Aussillous, P. \& Quéré, D. 2000 Quick deposition of a fluid on the wall of a tube. Physics of Fluids 12, 2367-2371.

Balestra, G., Zhu, L. \& Gallaire, F. 2017 A viscous droplet in a capillary tube: from Brethertons theory to empirical models. arXiv (1711.10447), 1-26.

Baroud, C., Gallaire, F. \& Dangla, R. 2010 Dynamics of microfluidic droplets. Lab on a chip 10, 2032-2045.

Bergeron, V. \& Radke, C. J. 1992 Equilibrium Measurements of Oscillatory Disjoining Pressures in Aqueous Foam Films. Langmuir 8 (19), 3020-3026.

Bico, J. \& QuÉRÉ, D. 2002 Self-propelling slugs. Journal of Fluid Mechanics 467, 101-127.

Bretherton, F. P. 1961 The motion of long bubbles in tubes. Journal of Fluid Mechanics 10 (02), 166-188.

Burgess, D. \& Foster, M. R. 1990 Analysis of the boundary conditions for a Hele-Shaw bubble. Physics of Fluids 2 (7), 1105-1117.

Cantat, I. 2013 Liquid meniscus friction on a wet plate: Bubbles, lamellae, and foams. Physics of Fluids 25, 31303-24904.

Chaudhury, K., Acharya, P. V. \& Chakraborty, S. 2014 Influence of disjoining pressure on the dynamics of steadily moving long bubbles inside narrow cylindrical capillaries. Physical Review E - Statistical, Nonlinear, and Soft Matter Physics 89 (5), 1-11.

Cohen-AdDAD, S. \& HöHleR, R. 2014 Rheology of foams and highly concentrated emulsions. Current Opinion in Colloid \& Interface Science 19 (6), 536-548.

Dangla, R., Kayi, S. C. \& Baroud, C. N. 2013 Droplet microfluidics driven by gradients of confinement. Proceedings of the National Academy of Sciences of the United States of America 110 (3), 853-858.

Denkov, N. D., Tcholakova, S., Golemanov, K., Ananthapadmanabhan, K. P. \& Lips, A. 2008 Viscous Friction in Foams and Concentrated Emulsions under Steady Shear. Physical Review Letters 100, 138301.

Derjaguin, B. V. 1993 On the Thickness of a Layer of Liquid Remaining on the Walls of Vessels after their Emptying, and the Theory of the Application of Photoemulsion after Coating on the Cine Film. Progress in Surface Science 43, 129-133.

Dias, E. O. \& Miranda, J. A. 2013 Control of centrifugally driven fingering in a tapered Hele-Shaw cell. Physical Review E 87 (053014).

EgGers, J. 2001 Air entrainment through free-surface cusps. Phys. Rev. Lett. 86, 4290-4293.

Eri, A. \& Okumura, K. 2011 Viscous drag friction acting on a fluid drop confined in between two plates. Soft Matter 7 (12), 5648-5653.

Gauglitz, P. A. \& Radke, C. J. 1990 The dynamics of liquid film breakup in constricted cylindrical capillaries. Journal of Colloid and Interface Science 134 (1), 14-40.

Hammoud, N. H., Trinh, P. H., Howell, P. D. \& Stone, H. A. 2017 Influence of van der waals forces on a bubble moving in a tube. Phys. Rev. Fluids 2, 063601.

Hodges, S. R., Jensen, O. E. \& Rallison, J. M. 2004 The motion of a viscous drop through a cylindrical tube. Journal of Fluid Mechanics 501, 279-301.

Homsy, G. M. 1987 Viscous fingering in porous media. Annual Review of Fluid Mechanics 19 (9), 271-311.

Huerre, A., Théodoly, O., Leshansky, A. M., Valignat, M.-P., Cantat, I. \& Jullien, M.-C. 2015 Droplets in Microchannels: Dynamical Properties of the Lubrication Film. Physical Review Letters 115, 064501.

IsRaelachvili, J. 2011 Intermolecular and Surface Forces. In Intermolecular and Surface Forces, 3rd edn. Elsevier.

Jung, M., Brinkmann, M., Seemann, R., Hiller, T., Sanchez De, M., Lama, L. \& Herminghaus, S. 2016 Wettability controls slow immiscible displacement through local interfacial instabilities. Physical Review Fluids 1, 074202.

Keiser, L., Herbaut, R., Bico, J. \& Reyssat, É. 2016 Washing wedges: capillary instability in a gradient of confinement. J. Fluid Mech 790, 619-633. 
Klaseboer, E., Gupta, R. \& Manica, R. 2014 An extended Bretherton model for long Taylor bubbles at moderate capillary numbers. Physics of Fluids 26 (19), 032107.

Landau, L. \& Levich, V. G. 1942 Dragging of a liquid by a moving plate. Acta Physicochimica USSR 17, 42.

Li, Y.-C., Liao, Y.-C., Wen, T.-C. \& Wei, H.-H. 2014 Breakdown of the Bretherton law due to wall slippage. Journal of Fluid Mechanics 741, 200-227.

Ling, Y., Fullana, J.-M., Popinet, S. \& Josserand, C. 2016 Droplet migration in a HeleShaw cell: Effect of the lubrication film on the droplet dynamics. Physics of Fluids 28, 062001.

Lorenceau, É., Restagno, F. \& Quéré, D. 2003 Fracture of a viscous liquid. Phys. Rev. Lett. 90, 184501.

Marchand, A., Chan, T. S., Snoeijer, J. H. \& Andreotti, B. 2012 Air entrainment by contact lines of a solid plate plunged into a viscous fluid. Phys. Rev. Lett. 108, 204501.

PArk, C.-W. \& Homsy, G. M. 1984 Two-phase displacement in hele shaw cells: theory. Journal of Fluid Mechanics 139, 291308.

Pihler-Puzovic, D., Illien, P., Heil, M. \& Juel, A. 2012 Suppression of Complex Fingerlike Patterns at the Interface between Air and a Viscous Fluid by Elastic Membranes. Phys. Rev. Lett. 108.

Preira, P., Valignat, M.-P., Bico, J. \& Théodoly, O. 2013 Single cell rheometry with a microfluidic constriction: Quantitative control of friction and fluid leaks between cell and channel walls. Biomicrofluidics 7 (2), 024111.

Princen, H. M. \& Kiss, A. D. 1989 Rheology of foams and highly concentrated emulsions. Journal of Colloid and Interface Science 128 (1), 176-187.

Reichert, B., Huerre, A., Théodoly, O., Valignat, M.-P. \& Jullien, M.-C. 2018 . to be published .

REYSSAT, É. 2014 Drops and bubbles in wedges. Journal of Fluid Mechanics 748, 641-662.

Saffman, P. G. \& TAYlor, G. I. 1958 Liquid The penetration of a fluid into a porous medium or Hele-Shaw cell containing a more viscous liquid. Proceedings of the Royal Society of London. Series A. Mathematical and Physical Sciences 245 (1242), 312-329.

Sauret, A., Boulogne, F., Cappello, J., Dressaire, E. \& Stone, H. A. 2015 Damping of liquid sloshing by foams. Physics of Fluids 27, 022103.

Schwartz, L. W., Princen, H. M. \& Kiss, A. D. 1986 On the motion of bubbles in capillary tubes. Journal of Fluid Mechanics 172, 259-275.

Seemann, R., Brinkmann, M., Pfohl, T. \& Herminghaus, S. 2012 Droplet based microfluidics. Reports on Progress in Physics 75, 016601.

Seiwert, J., Clanet, C. \& Quéré, D. 2011 Coating of a textured solid. Journal of Fluid Mechanics 669, 55-63.

Shen, B., Leman, M., Reyssat, M. \& Tabeling, P. 2014 Dynamics of a small number of droplets in microfluidic HeleShaw cells. Experiments in Fluids 55, 1728.

Snoeijer, J. H. \& Andreotti, B. 2013 Moving Contact Lines: Scales, Regimes, and Dynamical Transitions. Annu. Rev. Fluid Mech 45, 269-292.

Snoeijer, J. H., Delon, G., Fermigier, M. \& Andreotti, B. 2006 Avoided critical behavior in dynamically forced wetting. Phys. Rev. Lett. 96, 174504.

TAYlor, G. I. 1961 Deposition of a viscous fluid on the wall of a tube. Journal of Fluid Mechanics 10, 161-165.

Teletzke, G. F., Davis, H. T. \& Scriven, L. E. 1988 Wetting hydrodynamics. Revue de Physique Appliquée 23 (6), 989-1007.

Viola, F., Brun, P.-T., Dollet, B. \& Gallaire, F. 2016 Foam on troubled water: Capillary induced finite-time arrest of sloshing waves. Physics of Fluids 28, 091701.

Yahashi, M., Кimoto, N. \& Okumura, K. 2016 Scaling crossover in thin-film drag dynamics of fluid drops in the Hele-Shaw cell. Scientific Reports 6, 31395.

Zhao, B., Pahlavan, A. A., Cueto-Felgueroso, L. \& Juanes, R. 2018 Forced Wetting Transition and Bubble Pinch-Off in a Capillary Tube. Phys. Rev. Lett. 120, 084501.

Zhou, C., Yue, P. \& Feng, J. J. 2007 Simulation of neutrophil deformation and transport in capillaries using Newtonian and viscoelastic drop models. Annals of Biomedical Engineering 35 (5), 766-780. 
Zhu, L. \& Gallaire, F. 2016 A pancake droplet translating in a Hele-Shaw cell: lubrication film and flow field. Journal of Fluid Mechanics 798, 955-969.

Zinchenko, A. Z. \& Davis, R. H. 2017 Motion of Deformable Drops Through Porous Media. Annual Review of Fluid Mechanics 49 (1). 